\title{
Attenuation and dispersion of P-waves in porous rocks with planar fractures: Comparison of theory and numerical simulations
}

\author{
Gracjan Lambert ${ }^{1}$, Boris Gurevich ${ }^{2}$, and Miroslav Brajanovski ${ }^{1}$
}

\begin{abstract}
To explore the validity and limitations of the theoretical model of wave propagation in porous rocks with periodic distribution of planar fractures, we perform numerical simulation using a poroelastic reflectivity algorithm. The numerical results are found to be in good agreement with the analytical model, not only for periodic fractures, but also for random distribution of constant thickness fractures.
\end{abstract}

\section{INTRODUCTION}

Naturally fractured reservoirs have attracted an increased interest of exploration and production geophysics in recent years. In many instances, natural fractures control the permeability of the reservoir; hence, the ability to find and characterize fractured areas of the reservoir represents a major challenge for seismic studies.

One of the main issues in the characterization of any reservoir is the ability to predict the effect of fluid on its elastic properties. For isotropic porous reservoirs, this effect is expressed through Gassmann's (1951) equations, which provide explicit analytical expressions for the effective elastic moduli of a fluid-saturated rock as functions of the porosity, the elastic moduli of the dry skeleton, bulk modulus of the solid grain material, and the bulk modulus of the pore fluid. In fractured and porous reservoirs, the effect of the saturating fluid on elastic properties becomes more complex because the fluid affects the elastic anisotropy of the rock and also causes significant frequency dependent attenuation and dispersion (Thomsen, 1995; Hudson et al. 1996; 2001; Tod, 2001).

A more general approach to modeling the effect of fractures and fluids on elastic properties of porous media can be based on Biot's (1962) theory of poroelasticity. For a porous rock with aligned fractures, such an approach was recently developed by Brajanovski et al. (2003), who modeled fractures as thin and highly compliant layers in a porous background. Specifically, assuming that the porous medium is permeated by a periodic sequence of such fractures (layers) and using the results of Norris (1993) for frequency dependent effective moduli of a periodically layered poroelastic medium, Brajanovski et al. (2003) derived the dispersion equation for compressional waves propagating in the direction perpendicular to the fracture plane.

This model was limited by the assumptions of infinitely small fracture thickness and the spatial periodicity of fractures. Analysis of the sensitivity of attenuation and dispersion to these assumptions can be done using numerical simulations. In this paper, we perform such an analysis by modeling fractures as layers of small but finite thickness and high but finite porosity and simulate the propagation of seismic waves using a poroelastic reflectivity algorithm.

\section{THEORETICAL MODEL}

Consider a set of periodically alternating layers of two different porous materials, with subscript $b$ representing layers of a background porous rock, and subscript $c$ representing thin layers of a soft porous material (which in the limit of a vanishing volume fraction will become the fracture or crack). Both background and fractures are modeled by Biot's poroelasticity theory. Relevant material properties of the modeled media are the porosity $\phi$, permeability $\kappa$, dry (drained) bulk modulus $K^{\text {dry }}$, and shear modulus $\mu$. The spatial period of the system (fracture spacing) is $H$, and volume fractions are $h_{b}$ and $h_{c} \ll h_{b}$, where $h_{c}+h_{b}=1$. Background and fractures are assumed to consist of the same isotropic grain material of bulk modulus $K_{g}$, shear modulus $\mu_{g}$, and density $\rho_{g}$ and are saturated with the same fluid of bulk modulus $K_{f}$, density $\rho_{f}$, and dynamic viscosity $\eta$.

Norris (1993) and White et al. (1975) showed that for frequencies much smaller than Biot's characteristic frequency $\omega_{B}$ $=\eta \phi / \kappa \rho_{f}$, and also much smaller than the resonant frequency of

Manuscript received by the Editor May 21, 2004; revised manuscript received September 15, 2005; published online May 26, 2006.

Curtin University of Technology, Department of Exploration Geophysics, GPO Box U1987, Perth, Western Australia 6845, Australia. E-mail: Gracian.Lambert@geophy.curtain.edu.au; Miroslav.Brajanovski@geophy.curtain.edu.au.

${ }^{2}$ Curtin University of Technology, Department of Exploration Geophysics, GPO Box U1987, Perth, Western Australia 6845, Australia; and CSIRO Petroleum, GPO Box 1130, Bentley, Western Australia 6102, Australia. E-mail: Boris.Gurevich@ geophy.curtain.edu.au.

(C) 2006 Society of Exploration Geophysicists. All rights reserved. 
the layering, the compressional wave modulus $c_{11}^{\text {sat }}$ of P-waves propagating normal to a periodically layered, fluid-saturated porous medium composed of two constituents ( $b$ and $c$ ) can be written as

$$
\begin{aligned}
\frac{1}{c_{11}^{\text {sat }}}=\frac{h_{b}}{L_{b}^{\text {sat }}} & +\frac{h_{c}}{L_{c}^{\text {sat }}}+\frac{2}{\sqrt{i \omega \eta} H} \\
& \times \frac{\left(\frac{\sigma_{b} M_{b}}{L_{b}^{\text {sat }}}-\frac{\sigma_{c} M_{c}}{L_{c}^{\text {sat }}}\right)^{2}}{\sqrt{\frac{M_{b} L_{b}^{\text {dry }}}{L_{b}^{\text {sat }} \kappa_{b}}} \cot \left(\sqrt{\frac{i \omega \eta L_{b}^{\text {sat }}}{\kappa_{b} M_{b} L_{b}^{\text {dry }}}} \frac{h_{b} H}{2}\right)+\sqrt{\frac{M_{c} L_{c}^{\text {dry }}}{L_{c}^{\text {sat }} \kappa_{c}}} \cot \left(\sqrt{\frac{i \omega \eta L_{c}^{\text {sat }}}{\kappa_{c} M_{c} L_{c}^{\text {dry }}}} \frac{h_{c} H}{2}\right)} .
\end{aligned}
$$

In equation $1, \omega$ is the angular frequency, $L_{j}^{\text {dry }}=K_{j}^{\text {dry }}+4 \mu_{j} / 3$ is the plane deformation modulus of the dry material, and $L_{j}^{\text {sat }}$ is the saturated (undrained) modulus given by the Gassmann equation

$$
L_{j}^{\mathrm{sat}}=L_{j}^{\mathrm{dry}}+\sigma_{j}^{2} M_{j}
$$

with $M_{j}^{-1}=\left(\sigma_{j}-\phi_{j}\right) / K_{g}+\phi_{j} / K_{f}, \sigma_{j}=1-K_{j}^{\text {dry }} / K_{g}$, where indices $j=b$ and $j=c$ refer to background and fractures (cracks), respectively.

The effective P-wave modulus of the fractured porous medium can be obtained by taking the limit $h_{c} \rightarrow 0$ (and setting $h_{b}$ to 1 ) in equation 1 , while at the same time assuming $L_{c}$ and $\mu_{c}$ are $O\left(h_{c}\right)$ (and hence $K_{c}=O\left(h_{c}\right)$ and $\left.\sigma_{c} \rightarrow 1\right)$. In the dry case $\left(K_{f}=0\right)$, equation 1 yields the familiar Backus average:
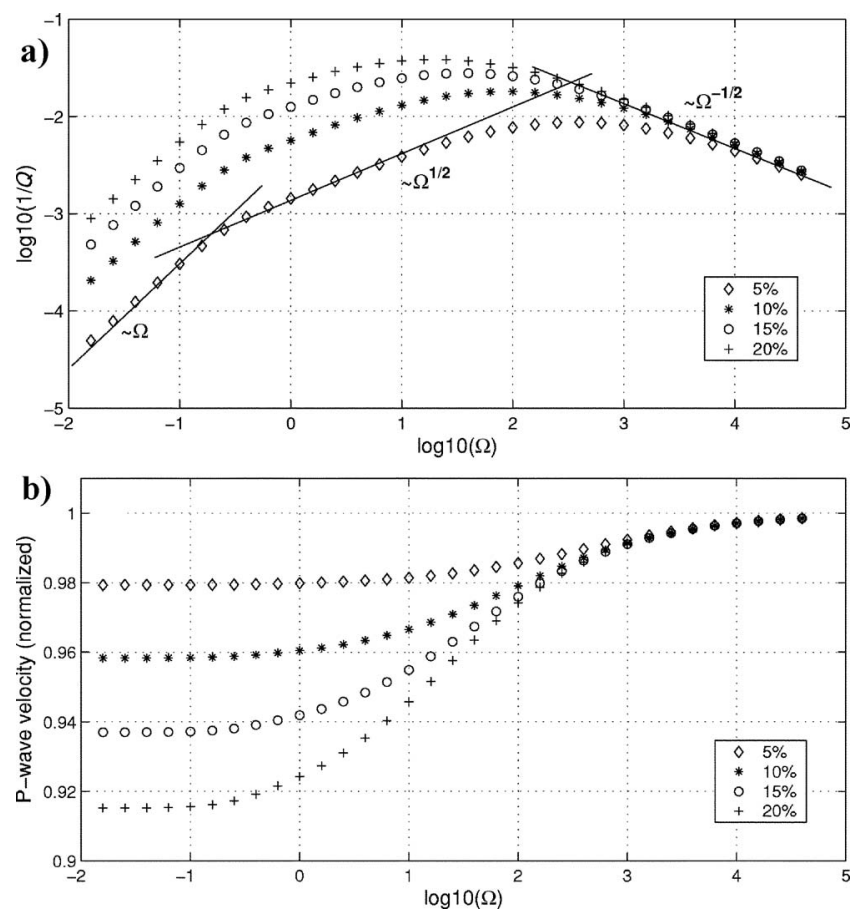

Figure 1. Normalized inverse quality factor $Q^{-1}$ (a) and P-wave velocity (b) as functions of normalized frequency $\Omega$ for rocks porosity $\phi=0.2$ with dry fracture weakness $\Delta_{N}$ ranging from 0.05 to 0.2 . Three different asymptotes can be identified for the attenuation curves.

$$
\frac{1}{c_{11}^{\mathrm{dry}}}=\frac{h_{b}}{L_{b}^{\mathrm{dry}}}+\frac{h_{c}}{L_{c}^{\mathrm{dry}}},
$$

where the limit $h_{c} \rightarrow 0$ yields

$$
\frac{1}{c_{11}^{\mathrm{dry}}}=\frac{1}{L_{b}^{\mathrm{dry}}}+Z_{N},
$$

and where

$$
Z_{N}=\lim _{h_{c} \rightarrow 0} \frac{h_{c}}{L_{c}^{\text {dry }}} .
$$

Equation 3 gives the stiffness $c_{11}$ of the elastic fractured medium, as described by the linear slip model of Schoenberg and Douma (1988), with normal excess fracture compliance $Z_{N}$ given by equation 4.

For the fluid-saturated rock taking the limit $\left(h_{c} \rightarrow 0\right.$ and $K_{c}^{\text {dry }}$ $\rightarrow 0$ ) in equation 1 yields the following dispersion equation for the plane compressional wave modulus $c_{11}^{\text {sat }}$ (Brajanovski et al., 2003):

$$
\frac{1}{c_{11}^{\text {sat }}}=\frac{1}{L_{b}^{\text {sat }}}+\frac{\Delta_{N}\left(\frac{\sigma_{b} M_{b}}{L_{b}^{\text {sat }}}-1\right)^{2}}{L_{b}^{\mathrm{dry}}\left[1-\Delta_{N}+\Delta_{N} \sqrt{i \Omega} \cot \left(\frac{L_{b}^{\mathrm{sat}}}{M_{b}} \sqrt{i \Omega}\right)\right]},
$$

where $\Delta_{N}=L_{b}^{\text {dry }} Z_{N} /\left(1+L_{b}^{\text {dry }} Z_{N}\right)$ is the so-called normal fracture weakness (Schoenberg and Douma, 1988), and $\Omega=\omega H^{2} \eta M_{b} /$ $4 \kappa_{b} L_{b}^{\text {sat }} L_{b}^{\text {dry }}$ is the normalized frequency. Equation 5 can be used to evaluate the frequency dependence of the P-wave velocity $V_{p}$ $=\left(\operatorname{Re} C_{11}^{\mathrm{sat}} / \rho_{b}\right)^{1 / 2}$ and attenuation (inverse quality factor $Q_{p}^{-1}$ $\left.=\left|\operatorname{Im} C_{11}^{\text {sat }} / \operatorname{Re} C_{11}^{\text {sat }}\right|\right)$, where $\rho_{b}=\rho_{g}\left(1-\phi_{b}\right)+\rho_{f} \phi_{b}$ is the density of the fluid-saturated background material. In the low-frequency limit, the velocity derived is consistent with anisotropic Gassmann equations (Gassmann, 1951; Brown and Korringa, 1975; Gurevich, 2003). In the high-frequency limit, the velocity in this direction is not affected by the fractures and is $V_{p}^{\infty}=\left(c_{11}^{b} / \rho_{b}\right)^{1 / 2}$.

Figure 1 shows dimensionless attenuation $Q^{-1}$ and velocity normalized by $V_{p}^{\infty}$ as a function of normalized frequency $\Omega$ for a water-saturated quartz sandstone with background porosity $\phi$ $=0.2$, permeability $\kappa=5 e-13 \mathrm{~m}^{2}$, frame moduli $K_{b}^{\text {dry }}=10 \mathrm{GPa}$ and $\mu_{b}=10 \mathrm{GPa}$, grain parameters $K_{g}=37 \mathrm{GPa}, \mu_{g}=44 \mathrm{GPa}$, and

$\rho_{g}=2.65 \mathrm{~g} / \mathrm{cm}^{-3}$, and variable fracture weakness. Velocity dispersion and attenuation curves have a typical shape for a relaxation phenomenon that is significant over a frequency range spanning at least two orders of magnitude. As expected, dispersion and attenuation increase with increased fracture weakness $\Delta_{N}$. For $\Delta_{N}=0.1$, the attenuation peak is observed at $\Omega=100$, which for fracture spacing $H=10 \mathrm{~m}$ corresponds to circular frequency of $f=2 \mathrm{kHz}$. As fracture weakness $\Delta_{N}$ increases, the attenuation peak shifts toward lower frequencies. In Figure 1a, three distinct asymptotes of attenuation can be identified. The low-frequency asymptote can be obtained by replacing the cotangent function in equation 5 with its Taylor expansion for a small argument. This yields attenuation proportional to frequency: $Q^{-1} \propto \Omega$. The high frequency asymptote is obtained by replacing the cotangent function with its limiting value for a large complex argument: 


$$
\lim _{\Omega \rightarrow \infty}\left[\cot \left(\frac{L_{b}^{\mathrm{sat}}}{M_{b}} \sqrt{i \Omega}\right)\right]=-i,
$$

which yields $Q^{-1} \propto \Omega^{-1 / 2}$. The middle asymptote, which is more pronounced for smaller values of $\Delta_{N}$, is obtained by first taking the limit $\Delta_{N} \rightarrow 0$ and then using the value defined by equation 6 for the cotangent. This yields power-law dependency $Q^{-1} \propto \Omega^{1 / 2}$ (Brajanovski, 2004).

\section{NUMERICAL MODELING}

The theoretical model described previously involves assumptions requiring infinitely small fracture thickness and the spatial periodicity of fractures. In order to gauge the influence of these limits on P-wave velocity dispersion and attenuation, we perform numerical modeling with fractures that are defined as layers of small but finite thickness and high but finite porosity.

Simulations of seismic wave propagation in layered media are best performed using reflectivity algorithms (Aki and Richards, 1980). In recent years, such algorithms have been adopted for poroelastic media (Allard et al., 1986). For our study, we employ the OASES global matrix algorithm developed by MIT (Schmidt and Tango, 1986) and adopted for poroelastic layers by Stern et al. (1985). OASES is an advanced 1.5D elastic reflectivity code that takes into account all the effects occurring in layered media (reflection, refraction, and mode conversion). For a given layered system, OASES computes the plane wave, complex transmission coefficient $T$. This coefficient then can be used to calculate the effective, complex velocity $V_{p}$ and attenuation $1 / Q$ in an equivalent homogeneous medium. For normal incidence, the transmission coefficient $T$ for such an equivalent medium can be written as

$$
T=\exp \left(i k H_{T}\right)=\exp \left(-\alpha H_{T}\right) \exp \left(i \omega H_{T} / V_{p}\right)
$$

where $H_{T}$ is the overall thickness of the layered system, $k=\omega / V_{p}$ $+i \alpha$ is the complex wavenumber of the transmitted $\mathrm{P}$-wave, and $\alpha$ is the effective attenuation coefficient. Taking absolute values of the left- and right-hand sides of equation 7 yields

$$
|T|=\exp \left(-\alpha H_{T}\right)
$$

or $\alpha=-\ln |T| / H_{T}$. Attenuation coefficient $\alpha$ is related to the inverse quality factor $1 / Q$ by (White, 1983)

$$
Q^{-1}=\frac{2 \alpha V_{p}}{\omega}=-\frac{2 V_{p}}{H_{T} \omega} \ln |T|
$$

Equation 9 can be used to compute the inverse quality factor from absolute values of the transmission coefficients obtained from OASES. Effective phase velocity in the stack of layers can be computed from the phase of the transmission coefficients obtained from OASES. Specifically, we obtain from OASES the phase $\Phi$ as the argument of transmission coefficient $T$, as given by equation 7 :

$$
\arg T=\frac{\omega H_{T}}{V_{p}} .
$$

Note that numerical values of phase as computed by OASES are wrapped. Once unwrapped, the phase can be used to compute the velocity from equation 10 .

\section{RESULTS}

The numerical technique described above is used to verify the theoretical results and to observe the influence of fracture distribution on attenuation and velocity dispersion. Simulations of wave propagation are performed on two types of models representing periodic and random fracture distribution. Both models utilize a total of 24 background layers with $20 \%$ porosity, alternating with 24 thin, high-porosity layers representing fractures. Simulations for both models were conducted using two fracture weakness values, 0.1 and 0.3 . The parameters of the background are taken to be the same as in the theoretical example given above. For every simulation, all layers were assumed to be saturated with water $\left(K_{f}\right.$ $\left.=2.25 \mathrm{GPa}, \rho_{f}=1.0 \mathrm{~g} / \mathrm{cm}^{-3}\right)$.

Figures $2 \mathrm{~b}$ and $2 \mathrm{c}$ show the attenuation and velocity for constant background layer thickness of $26.99 \mathrm{~m}$ and fracture thickness $0.01 \mathrm{~m}$ (Figure 2a). Fracture weakness is $\Delta_{N}=0.1$. This model represents a direct numerical equivalent of our theoretical model of periodic fracturing. We see that the numerical results agree very well with the theoretical prediction and that three distinct asymptotes $Q^{-1} \propto \Omega, Q^{-1} \propto \Omega^{1 / 2}$, and $Q^{-1} \propto \Omega^{-1 / 2}$ can be identified.

In order to investigate how critical the assumption of periodic (i.e., equidistant) fracture distribution is, we perform the same
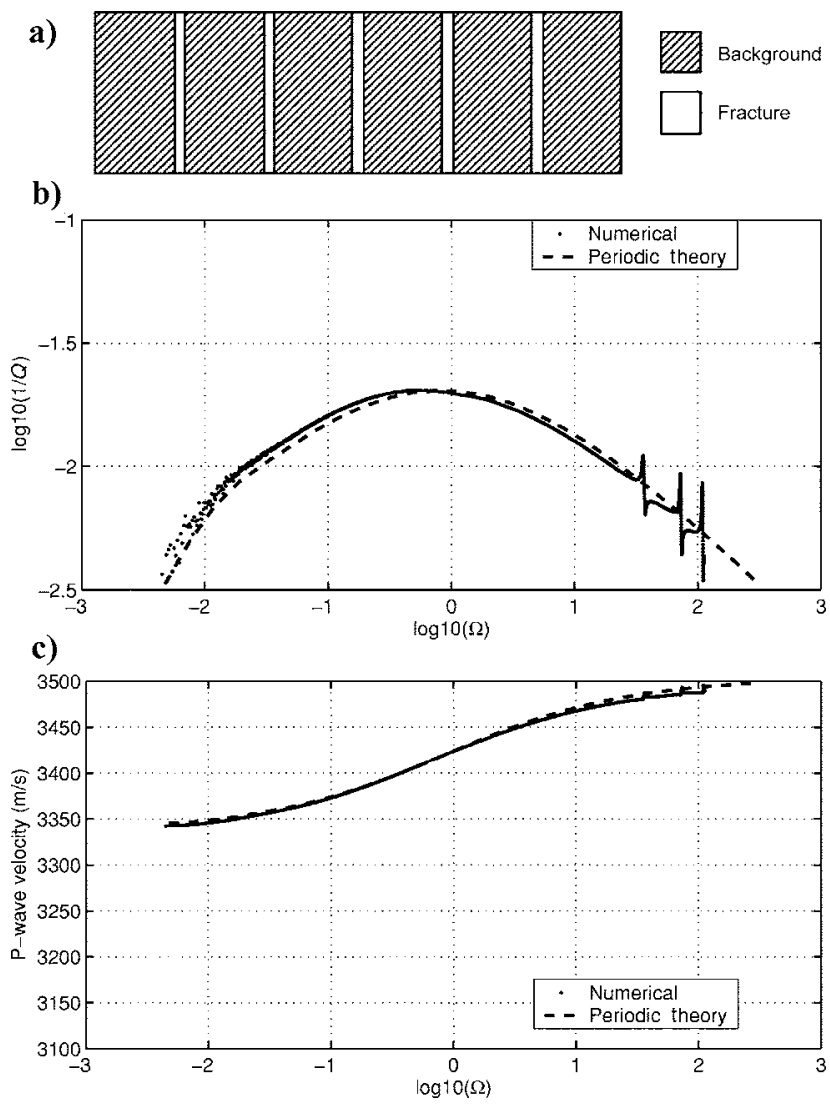

Figure 2. Periodic fracturing with a fracture weakness $\Delta_{N}=0.1$ : (a) cartoon representation, (b) attenuation, and (c) P-wave velocity versus normalized frequency $\Omega$. 
modeling for randomly spaced fractures. The random distribution of fractures is created by generating uniformly distributed random numbers in the interval $\left(0, H_{T}\right)$ and using them as $x_{1}$ coordinates of fracture locations (Figure 3a). These locations, together with the properties of the background and fractures, then are used as input parameters in OASES to compute the transmission coefficient, attenuation, and dispersion for a range of frequencies. Figures $3 \mathrm{~b}$ and $3 \mathrm{c}$ show the results for random fracture distribution (average background thickness of $26.99 \mathrm{~m}$, constant fracture thickness of 0.01 $\mathrm{m}$, and a fracture weakness $\left.\Delta_{N}=0.1\right)$ compared with our theoretical prediction (equation 5) for a periodic system of fractures. The agreement is still reasonably good; however, the low-frequency asymptote $Q^{-1} \propto \Omega^{1 / 2}$ is not identifiable. Instead, the middle asymptote $\Omega^{-1} \propto \Omega$ appears to extend further into the lower frequencies. Modeling for many realizations of the random medium and for a variable number of layers was found to yield very similar results.

In order to analyze the asymptotic behavior of attenuation at low frequencies in more detail, the computations need to be extended to still lower frequencies, leading to increasing numerical errors as transmission coefficient $T$ (equation 8 ) becomes very close to unity. Note that for small $\alpha H_{T}, \ln T \simeq T-1$, so that computation of $1 / Q$ using equation 9 effectively involves subtraction of two close numbers. On the other hand, Figure 1 shows that it is easier to analyze the low-frequency behavior of attenuation for larger values of fracture weakness $\Delta_{N}$, as in this case the low-frequency asymptote extends to higher frequencies and larger values of attenuation. To this end, we perform calculations for periodic and random fracture distributions with a fracture weakness of 0.3 (Figures 4 and 5). Figure 4 shows that numerical results agree well with the theoretical solution. In contrast, the results for random distribu-

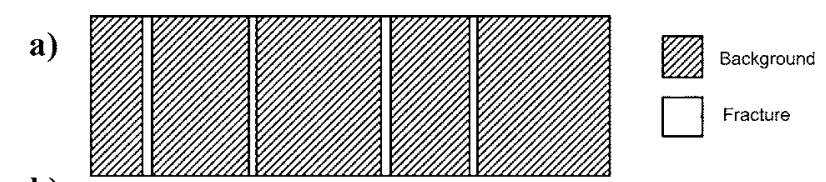

b)
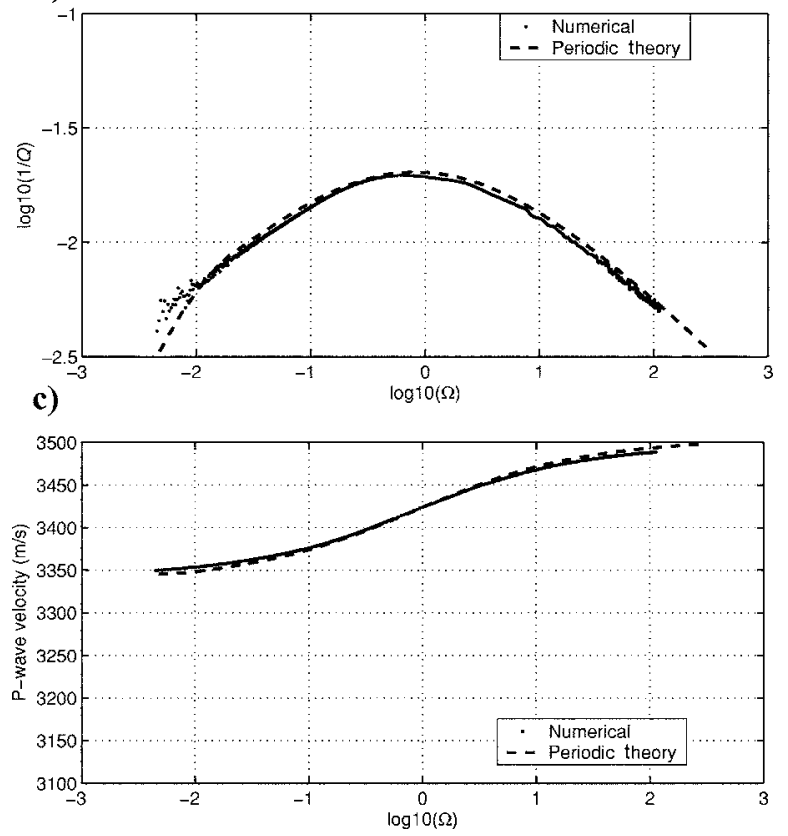

Figure 3. Same as Figure 2 but for random distribution of fractures.
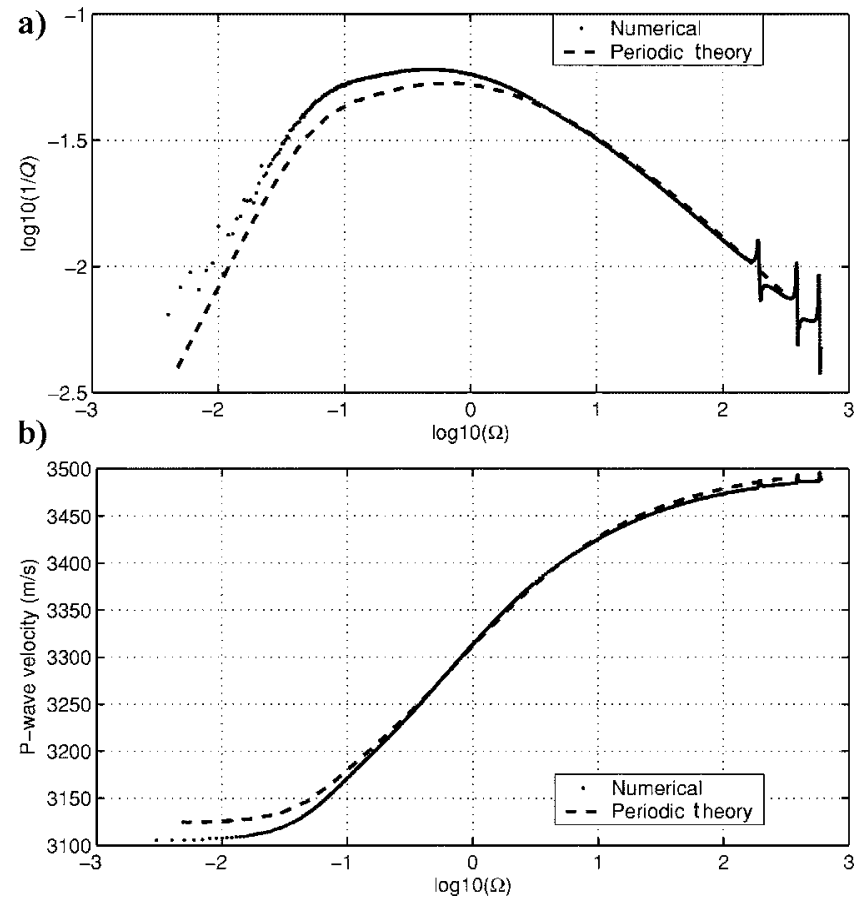

Figure 4. Same as Figure $2 \mathrm{~b}$ and $2 \mathrm{c}$ but for $\Delta_{N}=0.3$.
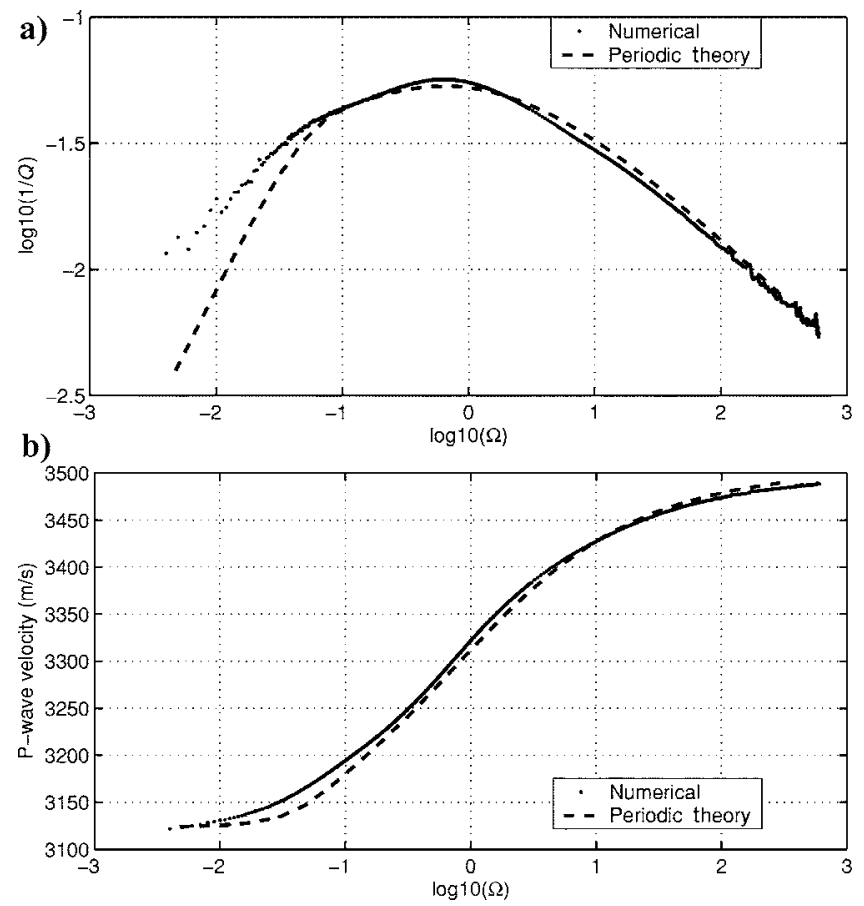

Figure 5. Same as Figure $3 \mathrm{~b}$ and $3 \mathrm{c}$ but for $\Delta_{N}=0.3$.

tion of fractures (Figure 5) clearly show that the low-frequency asymptote $Q^{-1} \propto \Omega$ is absent. Instead, attenuation is proportional to $\Omega^{1 / 2}$ in the low-frequency limit.

\section{DISCUSSION}

We have performed numerical simulations using the OASES re- 
flectivity code to explore the validity of the model of wave propagation in porous rocks with periodic fractures developed by Brajanovski et al. (2003). The two main results of this analysis are as follows. First, the numerical results for a periodic system of finitethickness fractures are in good agreement with the prediction of the analytical model. Second, the numerical results for a random system (uniform distribution) of fractures also show a reasonably good agreement with the analytical model for periodic fractures, especially in the vicinity of the attenuation peak. This latter finding appears to contradict earlier observations (Lopatnikov and Gurevich, 1988; Gurevic and Lopatnikov, 1995; Gurevich et al., 1997; Gelinsky, 1998) that show (both analytically and numerically) random and periodic systems of poroelastic layers exhibiting very different frequency dependencies of attenuation. However, our more detailed analysis, which involves a higher degree of fracturing (Figure 5), shows that periodic and random systems of fractures do exhibit different asymptotic behavior at low frequencies. This is consistent with the earlier studies cited above. These differences, nevertheless, may not have practical significance for most fractured rocks. As for most fractured rocks, as in most experiments, only relatively large values of attenuation around the peak are likely to be observed.

\section{CONCLUSION}

In summary, we can conclude that the theoretical model of Brajanovski et al. (2003) is applicable both to periodic and random systems of fractures. This model can be used to relate frequency dependencies of velocity and attenuation to the degree of fracturing and/or background porosity and permeability (Maultzsch et al., 2003).

The numerical analysis performed in this paper concerns attenuation and dispersion of $\mathrm{P}$-waves propagating perpendicular to fractures. It would be interesting to use the same methodology to analyze both $\mathrm{P}$ - and $\mathrm{S}$-waves propagating in other directions, and hence, to estimate frequency dependent anisotropy. Such an analysis, however, would not be straightforward because the explicit expressions analogous to equation 1 are not known for moduli $c_{13}^{\text {sat }}$ or $c_{33}^{\text {sat }}$ and must be the subject of a separate study.

\section{ACKNOWLEDGMENTS}

This work has been supported by the Petroleum Exploration Society of Australia (PESA), CSIRO Division of Petroleum Resources, Cooperative Research Centre for Mining Technology and Equipment (CMTE), and Centre of Excellence for Exploration and Production Geophysics (CEEPG). The authors thank Tobias Müller for fruitful discussions.

\section{REFERENCES}

Aki, K., and P. G. Richards, 1980, Quantitative seismology: Theory and methods: W. H. Freeman \& Company.

Allard, J. F., R. Bourdier, and C. Depollier, 1986, Biot waves in layered media: Journal of Applied Physics, 60, 1926-1929.

Biot, M. A., 1962, Mechanics of deformation and acoustic propagation in porous media: Journal of Applied Physics, 33, 1482-1948.

Brajanovski, M., 2004, Effects of fractures on seismic waves in poroelastic formations: Ph.D. dissertation, Curtin University of Technology.

Brajanovski, M., B. Gurevich, and G. Lambert, 2003, Attenuation and dispersion of compressional waves in porous rocks with aligned fractures, 73rd Annual International Meeting, SEG, Expanded Abstracts, 16201623.

Brown, R. J. S., and J. Korringa, 1975, On the dependence of the elastic properties of a porous rock on the compressibility of the pore fluid: Geophysics, 40, 608-616.

Gassmann, F., 1951, Uber die Elastizitat poroser Medien: Viertel: Vierteljahrsschrift der Naturforschenden Gesellschaft in Zurich, 96, 1-23.

Gelinsky, S., S. Shapiro, T. Müller, and B. Gurevich, 1998, Dynamic poroelasticity of thinly layered structures: International Journal of Solids and Structures, 35, 4739-4752.

Gurevich, B., 2003, Elastic properties of saturated porous rocks with aligned fractures: Journal of Applied Geophysics, 54, 203-218.

Gurevich, B., and S. L. Lopatnikov, 1995, Velocity and attenuation of elastic waves in finely layered porous rocks: Geophysical Journal International, 121, 933-947.

Gurevich, B., V. B. Zyrianov, and S. L. Lopatnikov, 1997, Seismic attenuation in finely layered porous rocks: Effects of fluid flow and scattering: Geophysics, 62, 319-324.

Hudson, J. A., E. Liu, and S. Crampin, 1996, The mechanical properties of materials with interconnected cracks and pores: Geophysical Journal International, 124, 105-112.

Hudson, J. A., T. Pointer, and E. Liu, 2001, Effective medium theories for fluid saturated material with aligned cracks: Geophysical Prospecting, 49, 509-522.

Lopatnikov, S. L., and B. Gurevich, 1988, Transformational mechanism of elastic wave attenuation in saturated porous media: Izvestija Academy of Sciences USSR: Physics of the Solid Earth, 24, 151-154.

Maultzsch, S., M. Chapman, E. Liu, and X. Li, 2003, Modelling frequencydependent seismic anisotropy in fluid-saturated rock with aligned fractures: Implication of fracture size estimation from anisotropic measurements: Geophysical Prospecting, 51, 381-392.

Norris, A. N., 1993, Low frequency dispersion and attenuation in partially saturated rocks: Journal of the Acoustical Society of America, 94, 359370

Schmidt, H., and G. Tango, 1986. Efficient global matrix approach to the computation of synthetic seismograms: Geophysics Journal of the Royal Astronomical Society, 84, 331-359.

Schoenberg, M., and J. Douma, 1988. Elastic-wave propagation in media with parallel fractures and aligned cracks: Geophysical Prospecting, 36, 571-590.

Stern, M., A. Bedford, and H. R. Millwater, 1985. Wave reflection from a sediment layer with depth-dependent properties: Journal of the Acoustical Society, 77, 1781-1788.

Thomsen, L., 1995, Elastic anisotropy due to aligned cracks in porous rock, Geophysical Prospecting, 43, 805-829.

Tod, S. R., 2001, The effects on seismic waves of interconnected nearly aligned cracks: Geophysical Journal International, 146, 249-263.

White, J. E., 1983. Underground sound: Application of seismic waves. Elsevier Sciences Publishing, Inc.

White, J. E., N. G. Mikhaylova, and F. M. Lyakhovitsky, 1975, Lowfrequency seismic waves in fluid saturated layered rocks: Physics of the Solid Earth, 11, 654-659. 